Mr. Killeffer's book contains an account of the scientific and technical developments of the chenical and associated industries in the United States during the past thirty years. He writes with a good inside knowledge of his subject, and every paragraph reflects the optimism which is behind the growth of industries in his country. He reviews the industries producing drugs, antibiotics, chemicals from petroleum, synthetic rubber, vitamins, chemicals for the protection of crops from insects, weeds and fungi, atomic energy and radioactive isotopes, plastics and metals.

Then he considers the problems of the future. $\mathrm{He}$ has no fear that the growth of population in the world will ever lead to famine because "American Technologies are going to other countries for nationals there to develop and utilize". He explains that we shall never be short of metals, sources of energy to work our factories, or food. First there will be great advances in agriculture by improving both the intensity and extent of cultivation and fertilization of our fields. But eventually we shall produce our protein foods in factories by growing yeasts and moulds. But we may have to grow our sugars and carbohydrates on farm land where we can utilize the energy of the sun. He considers that "We already know how to live well even when fields and farms shrink. Centuries before we will need them for that purpose, our industry is already operating processes that will free us from the necessity of farming fields for food. We can properly put our fears in a deep freezer".

Mr. Killeffer believes that the social and political effects will be that "Universal peace can be easily and surely realized by applying technology and science through human co-operation and collaboration to solve the basic human problems of nutrition, health and resources. We already know how to produce plenty of food and how to free people from the fear of hunger and from the load that sickness, disease and misery lay upon them. We can overcome these terrors if we apply throughout the world the techniques that we already know".

R. E. SLADE

\section{NEW METHODS IN ASTRONOMY}

Radio Astronomy

By Dr. J. L. Pawsey and Dr. R. N. Bracewell. (International Monographs on Radio.) Pp. $x+361+$ 23 plates. (Oxford : Clarendon Press; London: Oxford University Press, 1955.) 55s. net.

T HE invention of methods of producing and detecting electromagnetic waves of long wavelength which could signal information invisibly over long distances with no material medium was a remarkable outcome of the classical electrodynamics of the nineteenth century. Although radio has since then continued to attract the attention of physicists, especially for the knowledge it gives of the Earth's upper atmosphere and ionosphere, pure research interest in radio became dwarfed by comparison with that in atomic physics. Much effort, however, was devoted by radio physicists and engineers to the perfection of radio communication in its various forms, including the associated techniques of radar.

The astronomer has always held a unique place in science. In the interpretation of his observations he incorporates new aspects of fundamental scientific knowledge ranging from nuclear physics to cosmology. $\mathrm{He}$ observes phenomena in conditions impossible to attain in the laboratory. His inferences and speculations arouse widespread interest since they concern man's place in the universe. That radio should now be making a far-reaching contribution to astronomy is an unexpected development of recent years.

The explanation lies in the advance of radio tech. nology as well as in astrophysical phenomena at radio wave-lengths. With radio receivers of special design the temperatures of objects can be measured by the radiation emitted to an accuracy of the order of one degree. Thus the temperature of the Moon can be measured by its radio emission. Radio can also measure the temperatures of ionized atmospheres such as the solar corona and interstellar ionized hydrogen in the vicinity of hot stars. In addition, there are solar and cosmic radio sources of amazingly high intensity, the interpretation of which is a challenge to the astronomer and physicist. Another discovery of great importance is the line emission at $1,420 \mathrm{Mc} / \mathrm{s}$. $(21 \mathrm{~cm}$.) from neutral hydrogen, from which there is no visual emission. This spectral line enables not only the spatial distribution of hydrogen in the galaxy to be studied, but also the velocity by the Doppler displacement. A valuable aid in determ. ining the structure of the galaxy is thus provided. Radar techniques have been extended to the study of reflexions from meteor trails and echoes from the Moon. In these various ways radio has become the new tool of astronomy and has the advantage of being able to 'see' through terrestrial clouds and interstellar dust.

Dr. J. L. Pawsey is the leader of an Australian research group, in the Commonwealth Scientific and Industrial Research Organization, which during the past ten years has played a leading part in the development of this new branch of astronomy. In their new book, Dr. Pawsey and Dr. R. N. Bracewell have written a valuable survey and guide to the fundamentals of the subject. It is an excellent introduction for physicists wishing to learn both the radio principles and the astronomical background. Equally, it explains the radio methods to the astronomer, and astronomy to the radio physicist or engineer.

It is plainly not easy to plan a book covering such a wide field. The authors have approached their task by describing radio techniques and propagation through an ionized gas; then follows a chapter on visual solar phenomena and solar physics. It is not until p. 144, with the chapter on solar radio waves, that the detailed account of the radio astronomical observations begins. An initial perusal of the chapters on solar and cosmic radio waves may be advisable in order to appreciate more thoroughly the significance of parts of the previous chapters.

The whole book is clearly written and well illustrated. The authors have endeavoured to be impartial in choosing material from the now vast number of papers on radio astronomy. It is, of course, natural that any author should be most advantageously placed in describing the work of his own laboratory. The rate of progress in radio astronomy is so great that a book on the subject can never be completely up to date when it is published. Although inevitably there are omissions of some recent findings, this book by Dr. Pawsey and Dr. Bracewell admirably covers the basic work and fundamentals. 